\title{
Simultaneous Pell equations
}

\author{
by \\ PingZhi YuAN (Guangdong)
}

1. Introduction. In this paper, we investigate positive integer solutions $(x, y, z)$ of some special forms of the simultaneous Diophantine equations

$$
\left\{\begin{array}{l}
a x^{2}-b z^{2}=\delta_{1}, \\
c y^{2}-d z^{2}=\delta_{2},
\end{array}\right.
$$

where $a, b, c$ and $d$ are positive integers, $\delta_{1}$ and $\delta_{2}$ are integers such that $\operatorname{gcd}\left(a b, \delta_{1}\right)=\operatorname{gcd}\left(c d, \delta_{2}\right)=1$. By work of Thue [10] and Siegel [8], (1) has at most finitely many solutions if $\left(b, \delta_{1}\right) \neq k\left(d, \delta_{2}\right)$, where $k$ is an integer. Considering (1) as an elliptic equation $a c(x y)^{2}=\left(b z^{2}+\delta_{1}\right)\left(d z^{2}+\delta_{2}\right)$, one may apply the theory of linear forms in logarithms to effectively bound all solutions $(x, y, z)$ of (1) and we can study the solutions of (1) via the arithmetic elliptic curves. The usual way to solve (1) completely is to combine lower bounds for the linear forms in logarithms of algebraic numbers with techniques from computational Diophantine approximations. Anglin [2] devotes Section 4.6 of his textbook to the description of an algorithm for solving some special forms of (1). For elementary arguments in certain cases of (1), see Walsh [11, 12], Bennett and Walsh [5] and the author's [14].

For the special Diophantine equations

$$
x^{2}-a z^{2}=y^{2}-b z^{2}=1
$$

where $a$ and $b$ are distinct positive integers, Anglin [1] showed that (2) has at most one positive solution $(x, y, z)$ whenever $\max (a, b) \leq 200$. Bennett [4], sharpening work of Masser and Rickert [6], proved that (2) has at most 3 positive solutions. The author [13], by using a different gap principle (to ensure that solutions do not lie too close together), showed that (2) has at most 2 positive solutions $(x, y, z)$ provided that $\max (a, b)>1.4 \cdot 10^{57}$.

2000 Mathematics Subject Classification: Primary 11D09, 11D25; Secondary 11B39, 11J13, 11J86.

Key words and phrases: simultaneous Diophantine equations, Diophantine approximations, linear forms in logarithms, Lucas' sequences. 
In the present paper, we will study the more general simultaneous Pell equations (1). In Section 2, some general information on (1) with $\delta_{i} \in$ $\{ \pm 1, \pm 2, \pm 4\}, i=1,2$, is obtained. On the other hand, in Theorems 2.1 and 2.2 we precisely say when (1) has infinitely many integer solutions $(x, y, z)$. Section 3 is devoted to the simultaneous Pell equations

$$
a x^{2}-b z^{2}=c y^{2}-d z^{2}=1
$$

where $a, b, c$ and $d$ are positive integers with $b \neq d$. In Section 4 we obtain some similar results to those in Section 3, but on the simultaneous Pell equations

$$
x^{2}-a y^{2}=y^{2}-b z^{2}=1
$$

where $a$ and $b$ are positive integers.

In Section 5, by using the same idea as in [13], we apply a result of Baker and Wüstholz [3], namely a lower bound for linear forms in logarithms of three algebraic numbers, to prove the following main theorems of this paper. Denote by $N(a, b, c, d)$ and $N(a, b)$ the number of positive integer solutions of (3) and (4), respectively. We have:

TheOREM 1.1. Let $a, b, c$ and $d$ be positive integers with $b \neq d$ and $\max (a, b, c, d) \geq 1.16 \cdot 10^{59}$. Then $N(a, b, c, d) \leq 2$.

Theorem 1.1 is a generalization of Theorem 1.4 of [13]. For equations (4), we have similar results which slightly improve Theorem 7.1 of [4]:

THeOREM 1.2. Let $a$ and $b$ be positive integers with $a>3.31 \cdot 10^{35}$. Then $N(a, b) \leq 2$.

THEOREM 1.3. Let $a$ and $b$ be positive integers and let $x_{1}+y_{1} \sqrt{a}$ be the fundamental solution of $x^{2}-a y^{2}=1$ (i.e. $x_{1}$ and $y_{1}$ are the smallest positive integers satisfying $\left.x_{1}^{2}-a y_{1}^{2}=1\right)$. If $y_{1}>a^{0.005}$ and $a>6.4 \cdot 10^{2326}$, then $N(a, b) \leq 1$.

Of course, we can obtain similar results for other forms of equation (1). Since the method is essentially similar, we omit them here.

For positive integers $l>1, m>1$ and $a>1$, let $n(l, m)$ and $c(l, a)$ be integers with

$$
n(l, m)=\frac{\left(m+\sqrt{m^{2}-1}\right)^{2 l}-\left(m-\sqrt{m^{2}-1}\right)^{2 l}}{4 \sqrt{m^{2}-1}}
$$

and

$$
4 c(l, a)-1=\frac{(\sqrt{a}+\sqrt{a-1})^{l}-(\sqrt{a}-\sqrt{a-1})^{l}}{2 \sqrt{a-1}}, \quad l \equiv 3(\bmod 4) .
$$

Then the simultaneous Pell equations

$$
x^{2}-\left(m^{2}-1\right) z^{2}=y^{2}-\left(n(l, m)^{2}-1\right) z^{2}=1, \quad x, y, z \in \mathbb{Z}
$$


and

$$
a x^{2}-(a-1) z^{2}=c(l, a) y^{2}-(c(l, a)-1) z^{2}=1, \quad x, y, z \in \mathbb{Z}
$$

have two positive solutions

$$
\begin{aligned}
& (x, y, z)=(m, n(l, m), 1), \\
& (x, y, z)=\left(\frac{\left(m+\sqrt{m^{2}-1}\right)^{2 l}+\left(m-\sqrt{m^{2}-1}\right)^{2 l}}{2}, 2 n(l, m)^{2}-1,2 n(l, m)\right)
\end{aligned}
$$

and

$$
\begin{aligned}
& (x, y, z)=(1,1,1) \\
& (x, y, z)=\left(\frac{(\sqrt{a}+\sqrt{a-1})^{l}+(\sqrt{a}-\sqrt{a-1})^{l}}{2 \sqrt{a}}, 4 c(l, a)-3,4 c(l, a)-1\right),
\end{aligned}
$$

respectively. We call $\left(a_{1}, b_{1}, c_{1}, d_{1}\right)$ an equivalent form of $(a, b, c, d)$ if $\left(a_{1}, b_{1}, c_{1}, d_{1}\right)=\left(a / a_{0}^{2}, b / b_{0}^{2}, c / c_{0}^{2}, d / d_{0}^{2}\right)$, where $a_{0}, b_{0}, c_{0}, d_{0}$ are positive integers. We think a more general result is true.

Conjecture 1.1. Apart from

$$
(a, b, c, d)=\left(1, m^{2}-1,1, n^{2}(l, m)-1\right),(a, a-1, c(l, a), c(l, a)-1)
$$

and their equivalent forms, $N(a, b, c, d) \leq 1$.

Acknowledgments. The work was done at Leiden University when the author was a visiting scholar. He is pleased to thank the staff of the Mathematical Institute for their hospitality. He thanks the referee for providing him with many valuable suggestions.

2. General results. Let $\delta_{i} \in\{ \pm 1, \pm 2, \pm 4\}, i=1,2, a, b, c$, and $d$ be positive integers such that neither $a b$ nor $c d$ is a perfect square and $\operatorname{gcd}\left(a b, \delta_{1}\right)=\operatorname{gcd}\left(c d, \delta_{2}\right)=1$. In this section, we investigate the following more general simultaneous Pell equations

$$
\left\{\begin{array}{l}
a x^{2}-b z^{2}=\delta_{1}, \\
c y^{2}-d z^{2}=\delta_{2} .
\end{array}\right.
$$

To discuss solutions $(x, y, z)$ of $(5)$, without loss of generality, we may assume that both $a x^{2}-b z^{2}=\delta_{1}$ and $c y^{2}-d z^{2}=\delta_{2}$ are solvable in positive integers.

Definition 2.1. If $a x^{2}-b y^{2}=\delta, \delta \in\{ \pm 1, \pm 2, \pm 4\}$, is solvable in positive integers, let $x_{0} \sqrt{a}+y_{0} \sqrt{b}$ be the smallest value of $x \sqrt{a}+y \sqrt{b}$ such that $(x, y)$ is a positive solution of $a x^{2}-b y^{2}=\delta$. Then $x_{0} \sqrt{a}+y_{0} \sqrt{b}$ is said to be the smallest solution of this equation. 
Let $x_{0} \sqrt{a}+z_{0} \sqrt{b}$ and $y_{0} \sqrt{c}+z_{0}^{\star} \sqrt{d}$ be the smallest solutions of $a x^{2}-b z^{2}$ $=\delta_{1}$ and $c y^{2}-d z^{2}=\delta_{2}$, respectively. Put

$$
\begin{array}{ll}
\alpha=\frac{x_{0} \sqrt{a}+z_{0} \sqrt{b}}{\sqrt{\left|\delta_{1}\right|}}, & \beta=\frac{y_{0} \sqrt{c}+z_{0}^{\star} \sqrt{d}}{\sqrt{\left|\delta_{2}\right|}}, \\
\bar{\alpha}=\frac{x_{0} \sqrt{a}-z_{0} \sqrt{b}}{\sqrt{\left|\delta_{1}\right|}}, & \bar{\beta}=\frac{y_{0} \sqrt{c}-z_{0}^{\star} \sqrt{d}}{\sqrt{\left|\delta_{2}\right|}} .
\end{array}
$$

Define

$$
U_{l}= \begin{cases}\frac{\alpha^{l}-\bar{\alpha}^{l}}{\sqrt{4 b /\left|\delta_{1}\right|}} & \text { if } 2 \nmid l \text { or }\left(b, \delta_{1}\right) \neq(1,-1),(1,-4), \\ \frac{\alpha^{l}+\bar{\alpha}^{l}}{\sqrt{4 /\left|\delta_{1}\right|}} & \text { if } 2 \mid l \text { and }\left(b, \delta_{1}\right)=(1,-1) \text { or }(1,-4) .\end{cases}
$$

Similarly, define $U_{k}^{\prime}$ to be $\left(\beta^{k}-\bar{\beta}^{k}\right) / \sqrt{4 d /\left|\delta_{2}\right|}$ or $\left(\beta^{k}+\bar{\beta}^{k}\right) / \sqrt{4 /\left|\delta_{2}\right|}$. First we have:

Lemma 2.1 ([9]). Let $x_{1} \sqrt{a}+y_{1} \sqrt{b}$ be the smallest solution of $a x^{2}-b y^{2}=$ $\delta, \delta \in\{1,2,4\}$. Then every positive solution $(x, y)$ of this equation can be given by

$$
\frac{x \sqrt{a}+y \sqrt{b}}{\sqrt{|\delta|}}=\left(\frac{x_{1} \sqrt{a}+y_{1} \sqrt{b}}{\sqrt{|\delta|}}\right)^{n}, \quad n>0,
$$

with $2 \nmid n$ if $\min (a, b)>1$ or $(a, \delta) \neq(1,1),(1,4)$.

Recall that if $\left(b, \delta_{1}\right) \neq k\left(d, \delta_{2}\right)$, then (1) has only finitely many solutions. If $\left(b, \delta_{1}\right)=k\left(d, \delta_{2}\right)$ and (1) has infinitely many solutions, then $a x^{2}=k c y^{2}$. Therefore, without loss of generality we may assume that $k=1$. We have:

Theorem 2.1. Let $a, b$ and $c$ be positive integers, $\delta \in\{ \pm 1, \pm 2, \pm 4\}$ such that neither $a b$ nor $b c$ is a perfect square and $\operatorname{gcd}(a b c, \delta)=1$. Then the simultaneous Pell equations

$$
\left\{\begin{aligned}
a x^{2}-b z^{2} & =\delta \\
c y^{2}-b z^{2} & =\delta
\end{aligned}\right.
$$

have a positive integer solution $(x, y, z)$ if and only if each equation in (6) is solvable in positive integers and ac is a perfect square. Moreover, if the system has a positive integer solution, then it has infinitely many integer solutions $(x, y, z)$.

Proof. It suffices to prove that if each equation in (6) is solvable and $a c$ is a perfect square, then (6) have infinitely many integer solutions. Let $a=a_{0} a_{1}^{2}$ with $a_{0}$ square-free. Since $a c$ is a perfect square, $a_{0}$ is moreover the square-free part of $c$, say, $c=a_{0} c_{1}^{2}$. Let $x_{0}^{\prime} \sqrt{a_{0}}+z_{0}^{\prime} \sqrt{b}$ be the smallest 
solution of $a_{0} x^{2}-b z^{2}=\delta$. From Lemma 2.1 we have

$$
\frac{x_{0} \sqrt{a}+z_{0} \sqrt{b}}{\sqrt{|\delta|}}=\left(\frac{x_{0}^{\prime} \sqrt{a_{0}}+z_{0}^{\prime} \sqrt{b}}{\sqrt{|\delta|}}\right)^{m}, \quad \frac{y_{0} \sqrt{c}+z_{0}^{\star} \sqrt{b}}{\sqrt{|\delta|}}=\left(\frac{x_{0}^{\prime} \sqrt{a_{0}}+z_{0}^{\prime} \sqrt{b}}{\sqrt{|\delta|}}\right)^{n}
$$

for some positive integers $m$ and $n$. Put

$$
\frac{x_{t} \sqrt{a_{0}}+z_{t} \sqrt{b}}{\sqrt{|\delta|}}=\left(\frac{x_{0}^{\prime} \sqrt{a_{0}}+z_{0}^{\prime} \sqrt{b}}{\sqrt{|\delta|}}\right)^{m n t}, \quad t=1,2, \ldots
$$

Then $a_{1} \mid x_{t}$ and $c_{1} \mid x_{t}$. Hence $\left(x_{t} / a_{1}, x_{t} / c_{1}, z_{t}\right)$ is a positive integer solution of (6) for every $t=1,2, \ldots$.

More generally we have:

THEOREM 2.2. Let $a, b$ and $c$ be positive integers, $\delta$ a nonzero integer such that neither $a b$ nor bc is a perfect square and $\operatorname{gcd}(a b c, \delta)=1$. Then the simultaneous Pell equations

$$
\left\{\begin{array}{l}
a x^{2}-b z^{2}=\delta \\
c y^{2}-b z^{2}=\delta
\end{array}\right.
$$

have a positive integer solution $(x, y, z)$ only if each equation in $(7)$ is solvable in positive integers and ac is a perfect square. Moreover, if they have a positive integer solution, then they have infinitely many integer solutions $(x, y, z)$.

Proof. It suffices to prove the last assertion. Let $a=a_{0} a_{1}^{2}, c=a_{0} c_{1}^{2}$ with $a_{0}$ square-free, and let $(x, y, z)$ be a positive solution of (7). Then $x \sqrt{a}+z \sqrt{b}=y \sqrt{c}+z \sqrt{b}$. Let $x_{0}+y_{0} \sqrt{a_{0} b a_{1}^{2} c_{1}^{2}}$ be the fundamental solution of $x^{2}-a_{0} b a_{1}^{2} c_{1}^{2} y^{2}=1$. Put

$$
x_{t} \sqrt{a_{0}}+z_{t} \sqrt{b}=(x \sqrt{a}+z \sqrt{b})\left(x_{0}+y_{0} \sqrt{a_{0} b a_{1}^{2} c_{1}^{2}}\right)^{t}, \quad t=1,2, \ldots
$$

It is easy to see that $a_{1}\left|x_{t}, c_{1}\right| x_{t}$ and $\left(x_{t} / a_{1}, x_{t} / c_{1}, z_{t}\right)$ is a positive integer solution of ( 7 ) for every $t=1,2, \ldots$.

By Theorems 2.1 and 2.2, we may assume that $\left(b, \delta_{1}\right) \neq\left(d, \delta_{2}\right)$ throughout the paper. We have the following lemmas:

LEMMA $2.2([7])$. If $\left(b, \delta_{1}\right) \neq(1,-1),(1,-4)$ and $m \mid n$, or $n / m$ is an odd integer, then $U_{m} \mid U_{n}$.

LEMMA 2.3. Let $k_{0}, k_{1}, k_{2}$ and $q$ be positive integers with $k_{2}=2 q k_{1} \pm k_{0}$, $0 \leq k_{0} \leq k_{1}$. Then $U_{k_{2}} \equiv \pm U_{k_{0}}\left(\bmod U_{k_{1}}\right)$.

Proof. Note that $k_{0}$ and $k_{2}$ have the same parities. We divide the proof into two cases. 
CASE I: $2 \nmid k_{2}$ or $\left(b, \delta_{1}\right) \neq(1,-1)$ or $(1,-4)$. We have

$$
\frac{\alpha^{2 q k_{1}+k_{0}}-\bar{\alpha}^{2 q k_{1}+k_{0}}}{\sqrt{4 b /\left|\delta_{1}\right|}}-(\alpha \bar{\alpha})^{q k_{1}} \frac{\alpha^{k_{0}}-\bar{\alpha}^{k_{0}}}{\sqrt{4 b /\left|\delta_{1}\right|}}=\frac{\left(\alpha^{q k_{1}+k_{0}}+\bar{\alpha}^{q k_{1}+k_{0}}\right)\left(\alpha^{q k_{1}}-\bar{\alpha}^{q k_{1}}\right)}{\sqrt{4 b /\left|\delta_{1}\right|}}
$$

and

$$
\begin{aligned}
\frac{\alpha^{2 q k_{1}-k_{0}}-\bar{\alpha}^{2 q k_{1}-k_{0}}}{\sqrt{4 b /\left|\delta_{1}\right|}+(\alpha \bar{\alpha})^{q k_{1}-k_{0}}} & \frac{\alpha^{k_{0}}-\bar{\alpha}^{k_{0}}}{\sqrt{4 b /\left|\delta_{1}\right|}} \\
& =\frac{\left(\alpha^{q k_{1}-k_{0}}+\bar{\alpha}^{q k_{1}-k_{0}}\right)\left(\alpha^{q k_{1}}-\bar{\alpha}^{q k_{1}}\right)}{\sqrt{4 b /\left|\delta_{1}\right|}} .
\end{aligned}
$$

Thus $U_{k_{2}} \equiv \pm U_{k_{0}}\left(\bmod U_{k_{1}}\right)$.

CASE II: $2 \mid k_{2}$ and $\left(b, \delta_{1}\right)=(1,-1)$ or $(1,-4)$. If $2 \mid q$ or $2 \nmid k_{1}$, then $\frac{\alpha^{2 q k_{1} \pm k_{0}}+\bar{\alpha}^{2 q k_{1} \pm k_{0}}}{\sqrt{4 /\left|\delta_{1}\right|}}-(\alpha \bar{\alpha})^{q k_{1}} \frac{\alpha^{k_{0}}+\bar{\alpha}^{k_{0}}}{\sqrt{4 /\left|\delta_{1}\right|}}=\frac{\left(\alpha^{q k_{1} \pm k_{0}}-\bar{\alpha}^{q k_{1} \pm k_{0}}\right)\left(\alpha^{q k_{1}}-\bar{\alpha}^{q k_{1}}\right)}{\sqrt{4 /\left|\delta_{1}\right|}}$.

If $2 \nmid q$ and $2 \mid k_{1}$, then

$$
\frac{\alpha^{2 q k_{1} \pm k_{0}}+\bar{\alpha}^{2 q k_{1} \pm k_{0}}}{\sqrt{4 /\left|\delta_{1}\right|}}+(\alpha \bar{\alpha})^{q k_{1}} \frac{\alpha^{k_{0}}+\bar{\alpha}^{k_{0}}}{\sqrt{4 b /\left|\delta_{1}\right|}}=\frac{\left(\alpha^{q k_{1} \pm k_{0}}+\bar{\alpha}^{q k_{1} \pm k_{0}}\right)\left(\alpha^{q k_{1}}+\bar{\alpha}^{q k_{1}}\right)}{\sqrt{4 /\left|\delta_{1}\right|}} \text {. }
$$

Thus again $U_{k_{2}} \equiv \pm U_{k_{0}}\left(\bmod U_{k_{1}}\right)$.

Lemma 2.4. Let the notations be as above and $\left(b, \delta_{1}\right) \neq\left(d, \delta_{2}\right)$. Let $z_{1}$ be the smallest positive integer $z$ of the solutions $(x, y, z)$ of (5). Then $z_{1} \mid z$ for any solution $(x, y, z)$ of $(5)$.

Proof. Let $\left(x_{1}, y_{1}, z_{1}\right)$ be the positive solution of (5) with smallest positive integer $z$, and $(x, y, z)$ be any solution of (5). Then from the definitions of $U_{l}$ and $U_{k}^{\prime}$, we have

$$
z_{1}=U_{l_{1}}=U_{k_{1}}^{\prime}, \quad z=U_{l}=U_{k}^{\prime}
$$

for some positive integers $l, k, l_{1}$ and $k_{1}$. If $l_{1} \mid l$ and $l / l_{1}$ is odd, then $z_{1} \mid z$, whence $k_{1} \mid k$ and $k / k_{1}$ is odd. If $k_{1} \mid k$ and $k / k_{1}$ is odd, then $z_{1} \mid z$, whence $l_{1} \mid l$ and $l / l_{1}$ is odd.

Suppose that $z_{1} \nmid z$. By the above discussion there are positive integers $q_{1}, q, l_{0}$ and $l$ such that

$$
l=2 q_{1} l_{1} \pm l_{0}, \quad 0 \leq l_{0}<l_{1}, \quad k=2 q k_{1} \pm k_{0}, \quad 0 \leq k_{0}<k_{1} .
$$

By Lemma 2.3 we have $z \equiv U_{l} \equiv \pm U_{l_{0}}\left(\bmod U_{l_{1}}\right)$ and $z \equiv \pm U_{k_{0}}^{\prime}\left(\bmod U_{k_{1}}^{\prime}\right)$. Hence

$$
U_{l_{0}} \equiv \pm U_{k_{0}}^{\prime}\left(\bmod z_{1}\right)
$$

If $2 \mid l l_{1}$, then $\left(b, \pm \delta_{1}\right)=(1,-1)$ or $(1,-4)$, and $\alpha=(u+v \sqrt{a}) / 2 \geq$ $(3+\sqrt{5}) / 2$, where $u$ and $v$ are positive integers with $u^{2}-v^{2} a=4$. If $2 \nmid l l_{1}$, then $k_{0}$ is odd, and $k_{0} \leq k_{1}-2$, and $\alpha=(u \sqrt{a}+v \sqrt{b}) /\left|\delta_{1}\right| \geq(\sqrt{5}+1) / 2$, where $u$ and $v$ are positive integers with $u^{2} a-v^{2} b=\delta_{1}$. Hence in both 
cases we have $U_{l_{0}}<\frac{1}{2} U_{l_{1}}$. Similarly, $U_{k_{0}}^{\prime}<\frac{1}{2} U_{k_{1}}^{\prime}$. Hence $\max \left(U_{l_{0}}, U_{k_{0}}^{\prime}\right)<$ $\frac{1}{2} \max \left(U_{l_{1}}, U_{k_{1}}^{\prime}\right)=\frac{1}{2} z_{1}$, and so (8) holds if and only if $U_{l_{0}}=U_{k_{0}}^{\prime}$. Therefore equations (5) have a solution $\left(x_{0}, y_{0}, z_{0}\right)$ with $z_{0}=U_{l_{0}}=U_{k_{0}}^{\prime}<z_{1}$. This contradicts our assumption that $z_{1}$ is the smallest such solution, so $z_{1} \mid z$, which proves our lemma.

Thanks to Lemma 2.4, when considering the number of solutions $(x, y, z)$ of (5), without loss of generality, we may assume that $\left(a, c, b, d, \delta_{1}, \delta_{2}\right)=$ $\left(1,1, m^{2}-\delta_{1}, n^{2}-\delta_{2}, \delta_{1}, \delta_{2}\right)$ or $\left(m^{2}+\delta_{1}, n^{2}+\delta_{2}, 1,1,-\delta_{1},-\delta_{2}\right), \delta_{i} \in\{1,4\}$, $i=1,2$, or $\left(a, c, a-\delta_{1}, c-\delta_{2}, \delta_{1}, \delta_{2}\right), \delta_{i} \in\{ \pm 1, \pm 2, \pm 4\}, i=1,2$. We will keep this assumption hereafter, whereby (5) has a trivial solution.

3. Lemmas for $a x^{2}-b z^{2}=c y^{2}-d z^{2}=1$. Throughout this section we assume that $c>a>1, \alpha=\sqrt{a}+\sqrt{a-1}$ and $\beta=\sqrt{c}+\sqrt{c-1}$. Suppose that $(x, y, z)$ is a positive integer solution of $(3)$; then

$$
z=\frac{\alpha^{l}-\alpha^{-l}}{2 \sqrt{a-1}}=\frac{\beta^{k}-\beta^{-k}}{2 \sqrt{c-1}}
$$

for some positive odd integers $l$ and $k$. Since $c>a$, from (9) it is clear that

$$
\sqrt{\frac{c-1}{a-1}} \alpha^{l}>\beta^{k}>\alpha^{l}, \quad\left(\frac{\beta}{\alpha}\right)^{2}>\sqrt{\frac{c-1}{a-1}},
$$

so if $k>1$ and $l>1$, then $l>k$.

Let

$$
\Lambda=\frac{1}{2} \log \frac{c-1}{a-1}+l \log \alpha-k \log \beta
$$

Then (9) implies that

$$
0<\Lambda=\log \left(1-\beta^{-2 k}\right)-\log \left(1-\alpha^{-2 l}\right)<-\log \left(1-\alpha^{-2 l}\right)<\frac{\alpha^{2}}{\alpha^{2}-1} \alpha^{-2 l} .
$$

It follows that

$$
\log \Lambda<-2 l \log \alpha+\log \frac{\alpha^{2}}{\alpha^{2}-1} .
$$

Suppose that $N(a, c) \geq 3$. Let $\left(x_{i}, y_{i}, z_{i}\right)(i=1,2,3)$ be the first three positive solutions of (3), say,

$$
z_{i}=\frac{\alpha^{l_{i}}-\alpha^{-l_{i}}}{2 \sqrt{a-1}}=\frac{\beta^{k_{i}}-\beta^{-k_{i}}}{2 \sqrt{c-1}}
$$

for some positive integers $l_{i}$ and $k_{i}(i=1,2,3)$ with $1=k_{1}<k_{2}<k_{3}$ and $1=l_{1}<l_{2}<l_{3}$. By the same discussions as in the proof of Lemma 2.4, we have:

LEMMA 3.1. With the above notations, either $l_{2} \mid l_{3}$ and $k_{2} \mid k_{3}$, or $l_{3}=$ $2 q l_{2} \pm 1$ and $k_{3}=2 q_{1} k_{2} \pm 1$ for some positive integers $q$ and $q_{1}$. 
Proof. If $l_{2} \mid l_{3}$, then $z_{2} \mid z_{3}$, whence $k_{2} \mid k_{3}$. Conversely, if $k_{2} \mid k_{3}$, then $l_{2} \mid l_{3}$. Now if $l_{2} \nmid l_{3}$, then $k_{2} \nmid k_{3}$, and let

$$
l_{3}=2 q l_{2} \pm l_{0}, \quad 0<l_{0}<l_{2}, \quad k_{3}=2 q_{1} k_{2} \pm k_{0}, \quad 0<k_{0}<k_{2},
$$

for some positive integers $q, q_{1}, k_{0}$ and $l_{0}$. By the same argument as in the proof of Lemma 2.4, we have $l_{0}=k_{0}=1$, and the same plus or minus sign occurs by Lemma 2.3 (Case I).

LEMMA 3.2. If $k_{2} \neq 3$, then $l_{3}>3.5 \cdot l_{2} \beta$.

Proof. We assume first that $l_{2} \mid l_{3}$. By Lemma 3.1 we have $l_{3}=q l_{2}$, $k_{3}=q_{1} k_{2}$ for odd positive integers $q$ and $q_{1}$. Therefore

$$
\frac{z_{3}}{z_{2}}=\frac{U_{q l_{2}}}{U_{l_{2}}}=\frac{U_{q_{1} k_{2}}^{\prime}}{U_{k_{2}}^{\prime}},
$$

which implies that $q>q_{1}$. Considering these equations modulo $z_{2}^{2}$, we have

$$
q\left(a x_{2}^{2}\right)^{(q-1) / 2} \equiv q_{1}\left(c y_{2}^{2}\right)^{\left(q_{1}-1\right) / 2}\left(\bmod z_{2}^{2}\right) .
$$

Since $a x_{2}^{2} \equiv c y_{2}^{2} \equiv 1\left(\bmod z_{2}^{2}\right)$, we get $q \equiv q_{1}\left(\bmod z_{2}^{2}\right)$. Hence $q>z_{2}^{2}>\beta^{8}$ and

$$
l_{3}>l_{2} \beta^{8} .
$$

Next assume that $l_{2} \nmid l_{3}$. By Lemma 3.1 we have $l_{3}=2 q l_{2} \pm 1$ and $k_{3}=$ $2 q_{1} k_{2} \pm 1$ for some positive integers $q$ and $q_{1}$. From $z_{3}=U_{k_{3}}^{\prime}=U_{l_{3}}$ we have $q>q_{1}$. Note that $\beta^{2 k_{2}}=2 z_{2}^{2}(c-1)+1+2 y_{2} z_{2} \sqrt{c(c-1)}$, so

$$
z_{3}=U_{k_{3}}^{\prime} \equiv 2 c q_{1} y_{2} z_{2} \pm 1\left(\bmod 2 z_{2}^{2}(c-1)\right) \text {. }
$$

Similarly,

$$
z_{3}=U_{l_{3}} \equiv 2 a q x_{2} z_{2} \pm 1\left(\bmod 2 z_{2}^{2}(a-1)\right) .
$$

From (13) and (14) we get

$$
a q x_{2} \equiv c q_{1} y_{2}\left(\bmod z_{2}\right) .
$$

Since $a x_{2}^{2} \equiv c y_{2}^{2} \equiv 1\left(\bmod z_{2}^{2}\right)$, we have

$$
c q_{1}^{2} \equiv a q^{2}\left(\bmod z_{2}\right) .
$$

If $a q^{2} \neq c q_{1}^{2}$, then $c q^{2}>\max \left(c q_{1}^{2}, a q^{2}\right)>z_{2}>\beta^{4}+\beta^{2}+1$ and $l_{3}=2 q l_{2} \pm 1 \geq$ $3.5 \cdot l_{2} \beta$.

If $a q^{2}=c q_{1}^{2}$, then $a=a_{1}^{2} u, c=c_{1}^{2} u, q=c_{1} t, q_{1}=a_{1} t$, where $a_{1}, c_{1}, u, t$ are positive integers with $\operatorname{gcd}\left(a_{1}, c_{1}\right)=1$ and $a_{1}<c_{1}$. Since $u\left(a_{1}^{2} x_{2}^{2}-c_{1}^{2} y_{2}^{2}\right)=$ $a x_{2}^{2}-c y_{2}^{2}=(a-c) z_{2}^{2}=u\left(a_{1}^{2}-c_{1}^{2}\right) z_{2}^{2}$ and $\operatorname{gcd}\left(z_{2}, a_{1} c_{1} u x_{2} y_{2}\right)=1$, we see that

$$
a_{1} x_{2}+c_{1} y_{2}=r \xi^{2}, \quad c_{1} y_{2}-a_{1} x_{2}=s \eta^{2}, \quad \operatorname{gcd}(\xi, s \eta) \mid 2,
$$

where $r, s, \xi, \eta$ are positive integers such that $z_{2}=\xi \eta$ or $2 \xi \eta$ and $r s=$ $c_{1}^{2}-a_{1}^{2}$ or $4\left(c_{1}^{2}-a_{1}^{2}\right)$. Now, by $(15), a_{1} c_{1} u\left(a_{1} x_{2}-c_{1} y_{2}\right) t \equiv 0\left(\bmod z_{2}\right)$, and 
so $\left(c_{1} y_{2}-a_{1} x_{2}\right) t \equiv 0\left(\bmod z_{2}\right)$, hence $2 t \equiv 0(\bmod \xi)$. Therefore

$$
c q^{2}=c_{1}^{4} u t^{2} \geq \frac{c_{1}^{4} u}{4} \xi^{2} \geq \frac{c_{1}^{4} u}{16\left(c_{1}^{2}-a_{1}^{2}\right)}\left(a_{1} x_{2}+c_{1} y_{2}\right)>z_{2}
$$

and $l_{3} \geq 3.5 \cdot l_{2} \beta$. The lemma is proved.

If $k_{2}=3$, then $z_{2}=U_{3}^{\prime}=4 c-1$. However:

Lemma 3.3. If $k_{2}=3$ and $\beta>1000$, then $l_{3}>1.8 \cdot l_{2} \beta^{2 / 3}$.

Proof. The proof is similar to that of Lemma 3.2. If $3 \mid k_{2}$, let $k_{3}=2 q_{1}$ and $l_{3}=l_{2} q$ for some positive integers $q$ and $q_{1}$, so that

$$
q\left(a x_{2}^{2}\right)^{(q-1) / 2} \equiv q_{1}\left(c y_{2}^{2}\right)^{\left(q_{1}-1\right) / 2}\left(\bmod z_{2}^{2}\right) .
$$

Note that $z_{2}=4 c-1$, and $a x_{2}^{2} \equiv c y_{2}^{2} \equiv 1\left(\bmod z_{2}^{2}\right)$, so $q>z_{2}^{2}=(4 c-1)^{2}$ $>\beta^{4}$, and $l_{3}=q l_{2}>l_{2} \beta^{4}$. If $3 \nmid k_{3}$, let $k_{3}=6 q_{1} \pm 1$ and $l_{3}=2 q l_{2} \pm 1$ for some positive integers $q$ and $q_{1}$. We have

$$
z_{3} \equiv 2 c q_{1} y_{2} z_{2} \pm 1 \equiv 2 a q x_{2} z_{2} \pm 1\left(\bmod z_{2}^{2}\right),
$$

and so

$$
c q_{1} y_{2} \equiv a q x_{2}\left(\bmod z_{2}\right) .
$$

Since $z_{2}=4 c-1$, we get $l \geq 7$ and $a x_{2}^{2} \equiv c y_{2}^{2} \equiv 1\left(\bmod z_{2}^{2}\right)$. We thus have

$$
q_{1}^{2} \equiv 4 c q_{1}^{2} \equiv 4 a q^{2}\left(\bmod z_{2}\right)
$$

whereby it follows that $q^{2} \geq c / a>0.9 \beta^{2 / 3}(\beta>1000)$ and $l_{3}=2 l_{2} q \pm 1>$ $1.8 \cdot l_{2} \beta^{2 / 3}$. The lemma is proved.

4. Some lemmas for $x^{2}-a y^{2}=y^{2}-b z^{2}=1$. In this section we give some lemmas related to the simultaneous equations

$$
\left\{\begin{array}{l}
x^{2}-a y^{2}=1 \\
y^{2}-b z^{2}=1
\end{array}\right.
$$

By Lemma 2.4 we may assume that $\alpha=x_{1}+y_{1} \sqrt{a}$ and $\beta=y_{1}+\sqrt{y_{1}^{2}-1}$, where $b=y_{1}^{2}-1$. Suppose that $(x, y, z)$ is a positive integer solution of (4). Then

$$
y=\frac{\alpha^{l}-\alpha^{-l}}{2 \sqrt{a}}=\frac{\beta^{k}+\beta^{-k}}{2}
$$

for some positive odd integers $l$ and $k$. From (19) we have

$$
\sqrt{\frac{1}{a}} \alpha^{l}>\beta^{k}
$$

Hence if $k>1$ and $l>1$, then $k>l$.

Let

$$
\Lambda=l \log \alpha-k \log \beta-\frac{1}{2} \log a .
$$


Then (19) implies that

$$
0<\Lambda=\log \left(1+\beta^{-2 k}\right)+\log \left(1-\alpha^{-2 l}\right)<\bar{\beta}^{2 k}+\frac{\alpha^{2}}{\alpha^{2}-1} \alpha^{-2 l}<1.5 \cdot \bar{\beta}^{2 k} .
$$

Hence

$$
\log \Lambda<-2 k \log \beta+\log 1.5 .
$$

Suppose that $N_{1}(a, b) \geq 3$. Let $\left(x_{i}, y_{i}, z_{i}\right) \quad(i=1,2,3)$ be the first three positive solutions of (4), say,

$$
z_{i}=\frac{\alpha^{l_{i}}-\alpha^{-l_{i}}}{2 \sqrt{a}}=\frac{\beta^{k_{i}}+\beta^{-k_{i}}}{2}
$$

for some positive integers $l_{i}$ and $k_{i}(i=1,2,3)$ with $1=l_{1}<l_{2}<l_{3}$ and $1=k_{1}<k_{2}<k_{3}$. By the same arguments as in Section 3, we have:

LEMMA 4.1. With the above notations, either $l_{2} \mid l_{3}$ and $k_{2} \mid k_{3}$, or $l_{3}=$ $2 q l_{2} \pm 1$ and $k_{3}=2 q_{1} k_{2} \pm 1$ for some positive integers $q$ and $q_{1}$.

LEMMA 4.2. Suppose that $(x, y, z)$ is a positive integer solution of (18) and $y=U_{l}=V_{k}^{\prime}$. Then $l \equiv 1(\bmod 4)$ and $k>2 y_{1}^{2}$.

Proof. Note that from $\alpha^{2}=2 a y_{1}^{2}+1+2 x_{1} y_{1} \sqrt{a}$, we get $U_{l} / y_{1} \equiv l$ $\left(\bmod 4 y_{1}^{2}\right)$. Similarly, since $\beta^{2}=2 y_{1}^{2}-1+2 y_{1} \sqrt{y_{1}^{2}-1}$ we have $V_{k}^{\prime} / y_{1} \equiv$ $(-1)^{(k-1) / 2} k\left(\bmod 4 y_{1}^{2}\right)$. Therefore $l \equiv(-1)^{(k-1) / 2} k\left(\bmod 4 y_{1}^{2}\right)$, whence $k>$ $2 y_{1}^{2}$. The lemma is proved.

LEMMA 4.3. $k_{3}>5.5 \cdot k_{2} \alpha$.

Proof. We assume first that $l_{2} \mid l_{3}$. By Lemma 4.1 we have $l_{3}=q l_{2}$, $k_{3}=q_{1} k_{2}$ for odd positive integers $q$ and $q_{1}$. Therefore

$$
\frac{z_{3}}{z_{2}}=\frac{U_{q l_{2}}}{U_{l_{2}}}=\frac{U_{q_{1} k_{2}}^{\prime}}{U_{k_{2}}^{\prime}},
$$

which implies that $q_{1}>q$. By the same argument as in the proof of Lemma 4.2 we have

$$
q \equiv q_{1}(-1)^{\left(q_{1}-1\right) / 2}\left(\bmod 4 y_{2}^{2}\right) .
$$

Hence $q>2 y_{2}^{2}>2 \alpha^{8}$, so $k_{3}>2 k_{2} \alpha^{8}$.

Next assume that $l_{2} \nmid l_{3}$. By Lemma 4.1 we have $l_{3}=2 q l_{2} \pm 1$ and $k_{3}=$ $2 q_{1} k_{2} \pm 1$ for some positive integers $q$ and $q_{1}$. Similarly we have

$$
\begin{aligned}
y & =V_{k_{3}} \equiv(-1)^{q_{1}}\left(y_{1} \mp 2 q_{1} y_{2} z_{2}\left(y_{1}^{2}-1\right)\right)\left(\bmod 2 y_{2}^{2}\right), \\
z_{3} & =U_{l_{3}} \equiv \pm y_{1}+2 x_{1} x_{2} y_{2} q\left(\bmod 2 y_{2}^{2}\right) .
\end{aligned}
$$

Since $y_{1} \leq y_{2} / 2$, we get

$$
\mp(-1)^{q_{1}} q_{1} z_{2}\left(y_{1}^{2}-1\right) \equiv x_{1} x_{2} q\left(\bmod y_{2}\right) .
$$


Note that $l \neq 3$ by Lemma 4.2 . Since $x_{2}^{2} \equiv 1\left(\bmod y_{2}^{2}\right), z_{2}^{2}\left(y_{1}^{2}-1\right) \equiv-1$ $\left(\bmod y_{2}^{2}\right)$, we have

$$
q_{1}^{2}\left(y_{1}^{2}-1\right) \equiv x_{1}^{2} q^{2}\left(\bmod y_{2}\right) .
$$

Since $y_{1}^{2}-1$ is never a square of an integer when $y_{1}>1$, it follows that $\left(q_{1} y_{1}\right)^{2}$ or $\left(q x_{1}\right)^{2}>y_{2}>y_{1}\left(\alpha^{4}+\alpha^{2}+1\right)$. Hence $q_{1}>q>2.8 \cdot \alpha, k_{3}=$ $2 q_{1} k_{2} \pm 1>5.5 \cdot k_{2} \alpha$.

5. Proofs of the main results. First we recall the following famous result of Baker and Wüstholz [3]. Let $\alpha_{1}, \ldots, \alpha_{n}$ (with $n \geq 2$ ) denote algebraic numbers different from 0 and 1 . Let $K=Q\left(\alpha_{1}, \ldots, \alpha_{n}\right)$ and set $d=[K: Q]$. Define a modified height by the formula

$$
h_{m}(\alpha)=\max \{h(\alpha),|\log \alpha| / d, 1 / d\},
$$

where $h(\alpha)$ denotes the standard logarithmic Weil height of an algebraic number $\alpha$.

Theorem 5.1 (Baker-Wüstholz [3]). Let $b_{1}, \ldots, b_{n}$ be integers such that

$$
\Lambda=b_{1} \log \alpha_{1}+\cdots+b_{n} \log \alpha_{n} \neq 0 .
$$

Then if $B=\max \left\{\left|b_{1}\right|, \ldots,\left|b_{n}\right|\right\} \geq 3$, we have the inequality

$$
\log |\Lambda|>-C_{1} h_{m}\left(\alpha_{1}\right) \cdots h_{m}\left(\alpha_{n}\right) \log B
$$

with

$$
C_{1}=18(n+1) ! n^{n+1}(32 d)^{n+2} \log (2 n d) .
$$

Proof of Theorem 1.1. We apply Theorem 5.1 with

$$
\begin{gathered}
\alpha_{1}=(c-1) /(a-1), \quad \alpha_{2}=\alpha^{2}, \quad \alpha_{3}=\beta^{2}, \\
b_{1}=1, \quad b_{2}=l_{3}, \quad b_{3}=-k_{3},
\end{gathered}
$$

where $\alpha=\sqrt{a}+\sqrt{a-1}$ and $\beta=\sqrt{c}+\sqrt{c-1}$. Then

$$
\Lambda=\log \frac{c-1}{a-1}+l_{3} \log \alpha^{2}-k_{3} \log \beta^{2} .
$$

We may take $d=4$, and

$$
\begin{gathered}
h_{m}\left(\alpha_{1}\right)=\log (c-1)<2 \log \beta, \quad h_{m}\left(\alpha_{2}\right)=\log \alpha, \\
h_{m}\left(\alpha_{3}\right)=\log \beta, \quad B=l_{3} .
\end{gathered}
$$

Therefore by Theorem 5.1 we have

$$
\log |\Lambda|>-7.6420496 \cdot 10^{15} \log \alpha \log ^{2} \beta \log l_{3} .
$$

If $k_{2} \neq 3$, by Lemma $3.2,(11)$ and $(28)$,

$$
l_{3}<3.8210248 \cdot 10^{15} \log ^{3} l_{3} .
$$

It follows that $l_{3}<4.101 \cdot 10^{20}$. Hence by Lemma 3.2 and $l_{2} \geq 7$, we get

$$
c<7.1 \cdot 10^{38} \text {. }
$$


If $k_{3}=3$, similarly, by Lemma 3.3 , we obtain

$$
c<1.16 \cdot 10^{59} \text {. }
$$

Proof of Theorem 1.2. We apply Theorem 5.1 with

$$
\begin{gathered}
\alpha_{1}=\sqrt{a}, \quad \alpha_{2}=\alpha, \quad \alpha_{3}=\beta, \\
b_{1}=-1, \quad b_{2}=-l_{3}, \quad b_{3}=k_{3}, \quad n=3,
\end{gathered}
$$

where $\alpha=x_{1}+y_{1} \sqrt{a}$ and $\beta=y_{1}+\sqrt{y_{1}^{2}-1}$. Then

$$
\Lambda=-\frac{1}{2} \log a-l_{3} \log \alpha+k_{3} \log \beta .
$$

Take $d=4$, and

$$
\begin{gathered}
h_{m}\left(\alpha_{1}\right)=\frac{1}{2} \log a<\log \alpha, \quad h_{m}\left(\alpha_{2}\right)=\frac{1}{2} \log \alpha, \\
h_{m}\left(\alpha_{3}\right)=\frac{1}{2} \log \beta, \quad B=k_{3} .
\end{gathered}
$$

By Theorem 5.1 we have

$$
\log |\Lambda|>-9.56 \cdot 10^{14} \log ^{2} \alpha \log \beta \log k_{3} .
$$

On the other hand, by (22),

$$
\log |\Lambda|<-2 k_{3} \log \beta+\log 1.5 .
$$

By Lemma 4.3, (29) and (30) we get

$$
k_{3}<4.78 \cdot 10^{14} \log ^{3} k_{3} .
$$

It follows that $k_{3}<4.43 \cdot 10^{19}$. Hence by Lemma 4.3 and $k_{2} \geq 7$, we obtain

$$
a y_{1}^{2}<3.31 \cdot 10^{35} \text {. }
$$

Proof of Theorem 1.3. Similarly, by Lemma 4.2, (29) and (30) and the assumptions we have

$$
k_{2}<4.78 \cdot 10^{18} \log ^{3} k_{2} .
$$

It follows that $k_{2}<8 \cdot 10^{13}$. Hence by Lemma 4.2 ,

$$
a_{1}<6.34 \cdot 10^{2326} \text {. }
$$

This completes the proof.

\section{References}

[1] W. S. Anglin, Simultaneous Pell equations, Math. Comp. 65 (1996), 355-359.

[2] - , The Queen of Mathematics: An Introduction to Number Theory, Kluwer, Dordrecht, 1995.

[3] A. Baker and G. Wüstholz, Logarithmic forms and group varieties, J. Reine Angew. Math. 442 (1993), 19-62.

[4] M. A. Bennett, On the number of solutions of simultaneous Pell equations, ibid. 498 (1997), 173-200.

[5] M. A. Bennett and P. G. Walsh, Simultaneous Pell equations with few or no solutions, Indag. Math. 11 (2000), 1-12. 
[6] D. W. Masser and J. H. Rickert, Simultaneous Pell equations, J. Number Theory 61 (1996), 52-66.

[7] P. Ribenboim and W. L. McDaniel, The square terms in Lucas sequences, ibid. 58 (1996), 104-123.

[8] C. L. Siegel, Über einige Anwendungen diophantischer Approximationen, Abh. Preuss. Akad. Wiss. 1929, no 1.

[9] Q. Sun and P. Z. Yuan, On the diophantine equations $\left(a x^{n}-1\right) /(a x-1)=y^{2}$ and $\left(a x^{n}+1\right) /(a x+1)=y^{2}$, J. Sichuan Univ. Nat. Sci. Ed. 26 (1989), Special Issue, 20-24 (in Chinese with English summary).

[10] A. Thue, Über Annäherungswerte algebraischer Zahlen, J. Reine Angew. Math. 135 (1909), 284-305.

[11] P. G. Walsh, On integer solutions to $x^{2}-d y^{2}=1, z^{2}-2 d y^{2}=1$, Acta Arith. 82 (1997), 69-76.

[12] -, On two classes of simultaneous Pell equations with no solutions, Math. Comp. 68 (1999), 385-388.

[13] P. Z. Yuan, On the number of solutions of simultaneous Pell equations, Acta Arith. 101 (2002), 215-221.

[14] - On the number of solutions of $x^{2}-4 m(m+1) y^{2}=y^{2}-b z^{2}=1$, Proc. Amer. Math. Soc. 132 (2004), 1561-1566.

Department of Mathematics

Sun Yat-Sen University

Guangdong, Guangzhou 510275, P.R. China

E-mail: mcsypz@zsu.edu.cn

yuanpz@mail.csru.edu.cn

Received on 30.9.2002

and in revised form on 15.7 .2004 